\title{
A Perturbative Method for Calculating the Impedance of Coils on Laminated Ferromagnetic Cores
}

\author{
Serguei Maximov, Allen A. Castillo, Vicente Venegas, \\ José L. Guardado, and Enrique Melgoza
}

Program of Postgraduates and Research in Electrical Engineering, Instituto Tecnológico de Morelia, Avenida Tecnológico 1500 Col. Lomas de Santiaguito, 58120 Morelia, MICH, Mexico

Correspondence should be addressed to Serguei Maximov, sgmaximov@yahoo.com.mx

Received 24 February 2010; Revised 11 August 2010; Accepted 6 September 2010

Academic Editor: Francesco Pellicano

Copyright (C) 2010 Serguei Maximov et al. This is an open access article distributed under the Creative Commons Attribution License, which permits unrestricted use, distribution, and reproduction in any medium, provided the original work is properly cited.

\begin{abstract}
A new set of formulas for calculating the self and mutual impedances of coils on straight and closed laminated ferromagnetic cores of circular cross-section has been derived. The obtained formulas generalize the well-known formulas for impedances of coils on homogeneous ferromagnetic cores, for the case of laminated cores, and improve the previously known formulas for laminated cores. The obtained formulas are fully consistent with Maxwell's equations and, therefore, offer an excellent accuracy. The perturbation theory and the average field technique are used to solve Maxwell's equations inside and outside the core. The solution inside the core can also be used in the analysis of thermal effects occurring inside the laminated core.
\end{abstract}

\section{Introduction}

Analytic calculation of the self and mutual impedances for coils on ferromagnetic cores is not a new topic [1-7]. A rigorous analytic solution of Maxwell's equations with the relevant boundary conditions is the natural basis for deriving accurate formulas for the coil impedances. In this approach, eddy currents induced into a laminated core and the frequency dependence of the impedance due to skin effect are properly represented. The mathematical complexity of the approach is related with the geometry and structure of the ferromagnetic core, so, rigorous analysis of a realistic case may become complicated. Nevertheless, the problem may be solved to any required degree of accuracy by using different approximate techniques and modern computational methods. 
In the paper, an analytic study of electromagnetic field in a transformer with an infinitely long laminated ferromagnetic core of circular cross-section is presented. The formula for self and mutual impedance for filamentary turns is derived. Due to the laminated structure of the ferromagnetic core, some of the parameters involved in the Maxwell's equations, such as magnetic permeability, permittivity, and electrical conductance, are discontinuous functions. As a result, the electromagnetic field becomes discontinuous, it loses the axial symmetry, and the use of numerous boundary conditions on the surface between each lamination and dielectric is required. The problem becomes too complicated.

In this paper, a new approach to the impedance calculation for transformers, which considers the laminated core as a whole, is presented. In order to simplify the problem, preserving a good accuracy, the use of the average electromagnetic field is proposed. In this approach, the problem becomes similar to the case of a uniform but anisotropic ferromagnetic core, where the magnetic permeability, permittivity, and electrical conductance are slightly different in the $X$ and $Y$ directions. In turn, it means that there exists a small parameter which can be taken, for example, as the relation between the difference of the magnetic permeability in the $Y$ and $X$ directions and the magnetic permeability of the uniform ferromagnetic core. Then, Maxwell's equations can be solved by employing the perturbation theory $[8,9]$. In this approach, every function involved in Maxwell's equations is considered as a power series in the small parameter. The leading term of this series is the solution of the exactly solvable problem with the axial symmetry. The higher-order terms are the corrections that complement the leading term to the full solution of Maxwell's equations. An approximate solution of the full problem is obtained by truncating the series, keeping only the first two terms of the power series. The higher orders become successively less important, and, therefore, they can be neglected.

\section{Equations for the Average Electromagnetic Field}

In Figure 1, a ferromagnetic laminated core with filamentary turns is presented. The ferromagnetic core is taken to be infinitely long; each lamination is of permeability $\mu_{2}$ and conductivity $\sigma_{2}$. The laminations are separated by a dielectric of permittivity $\varepsilon_{2}$. The medium around the core is of permittivity $\varepsilon_{1}$ and permeability $\mu_{1}$. The core radius is $R$. An energizing coil of $N$ filamentary turns of radius $r_{1}$, placed at the coordinates $z=n \tau$, is considered. The ferromagnetic lamination width is $a$, and the distance between two near laminations is $b$.

The permeability, permittivity, and electrical conductance can be modeled by introducing the function $\Delta(x)$ presented in Figure 2, such that $\Delta(x)=1$ inside and $\Delta(x)=$ 0 outside the ferromagnetic lamination. Once the function $\Delta(x)$ is defined, the electrical conductance in the whole space can be represented in the following form:

$$
\sigma(x, y)=\sigma_{2} \theta(R-r) \Delta(x)
$$

where $\theta(R-r)$ is the Heaviside step function [10], such that $\theta(R-r)=1$ inside and $\theta(R-r)=0$ outside the core (the medium 1 is nonconducting).

The permittivity in terms of the function $\Delta(x)$ can be modeled as follows:

$$
\varepsilon(x, y)=\theta(R-r)\left[\varepsilon_{2}(1-\Delta(x))+\varepsilon_{1} \Delta(x)\right]+\varepsilon_{1} \theta(r-R),
$$




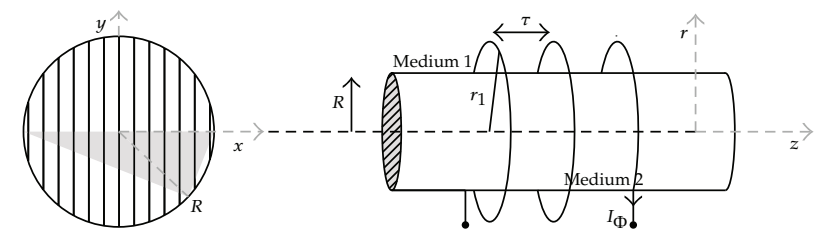

Figure 1: Filamentary turns on an infinite ferromagnetic core.

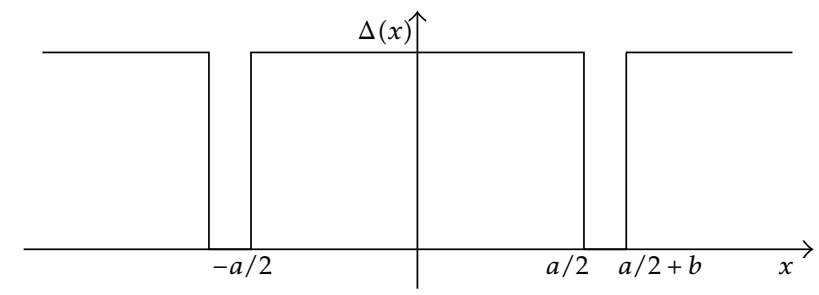

Figure 2: Function $\Delta(x)$.

and the permeability takes the form:

$$
\mu(x, y)=\theta(R-r)\left[\mu_{1}(1-\Delta(x))+\mu_{2} \Delta(x)\right]+\mu_{1} \theta(r-R)
$$

The mutual impedance between the energizing turn and a second filamentary turn of radius $r$, as well as the self impedance of a nonfilamentary turn of small cross-section, follows directly from the solution for the electric field intensity. We assume that the electromagnetic field is quasistationary, and all capacitive effects may be taken into account separately.

As it has been mentioned before, the problem has not cylindrical symmetry. Moreover, due to unhomogeneities of the ferromagnetic core, the structure of Maxwell's equations becomes complicated. In order to avoid such difficulty, we consider the electromagnetic field averaged with respect to the $X$ direction over $l=a+b$. The procedure of transforming the exact electromagnetic fields to the average fields comprises having on hand, for example, a bump function $p(\xi)$ [9], that is, compactly supported smooth (in the sense of $p(\xi) \in C^{\infty}$ ) function, such that

(a) $p(\xi) \geq 0$ for $0 \leq \xi \leq l$;

(b) $p(\xi)=0$ for $\xi<0$ and $\xi>l$;

(c) $\int_{-\infty}^{+\infty} p(\xi) d \xi=1$.

Let $\mathrm{f}(x, y, z)$ be one of the components of the electromagnetic field. Then, the averaged component $\langle f(x, y, z)\rangle$ is obtained as the following integral:

$$
\langle f(x, y, z)\rangle=\int_{-\infty}^{+\infty} f(x+\xi, y, z) p(\xi) d \xi .
$$


As a result, the averaged function $\langle f(x, y, z)\rangle$ has derivatives of all orders

$$
\frac{d^{n}}{d x^{n}}\langle\mathrm{f}(x, y, z)\rangle=(-1)^{n} \int_{-\infty}^{+\infty} \mathrm{f}(x+\xi, y, z) \frac{d^{n}}{d \xi^{k}} p(\xi) d \xi
$$

The averaged Maxwell's equations in the quasistationary approximation together with the continuity equation take the form

$$
\begin{gathered}
\nabla \times\langle\mathbf{H}\rangle=\langle\sigma \mathbf{E}\rangle+\langle\mathbf{j}\rangle, \\
\nabla \times\langle\mathbf{E}\rangle=-\left\langle\mu \frac{\partial \mathbf{H}}{\partial t}\right\rangle, \\
\nabla \cdot\langle\varepsilon \mathbf{E}\rangle=\langle\rho\rangle, \\
\nabla \cdot\langle\mu \mathbf{H}\rangle=0 \\
\frac{\partial\langle\rho\rangle}{\partial t}+\nabla \cdot\langle\sigma \mathbf{E}\rangle=0,
\end{gathered}
$$

where the averaged current density $\langle\mathbf{j}\rangle$ in the energizing coil is modeled by using the Dirac delta-function [10] as follows:

$$
\langle\mathbf{j}\rangle=(-y, x, 0) \frac{I_{\phi}}{r} \delta\left(r-r_{1}\right) \sum_{n=1}^{N} \delta(z-n \tau),
$$

where $r=\sqrt{x^{2}+y^{2}}$. The energizing filamentary turn carries a sinusoidal current $I_{\phi}(t)=$ $I_{\phi} e^{i \omega t}$.

The system of (2.6) is not closed due to the presence of the correlation functions $\langle\sigma \mathbf{E}\rangle$, $\langle\varepsilon \mathbf{E}\rangle$, and $\langle\mu \mathbf{H}\rangle$. In the medium 1, the parameters $\sigma=0, \varepsilon=\varepsilon_{1}, \mu=\mu_{1}$ are constants. Then, these correlation functions take the following simple form: $\langle\sigma \mathbf{E}\rangle=0,\langle\varepsilon \mathbf{E}\rangle=\varepsilon_{1}\langle\mathbf{E}\rangle,\langle\mu \mathbf{H}\rangle=$ $\mu_{1}\langle\mathbf{H}\rangle$. In the medium 2, the correlation functions are to be estimated.

Let us approximate the bump function $p(\xi)$ by the following discontinuous function:

$$
p(\xi)= \begin{cases}\frac{1}{l} & \text { for } 0 \leq \xi \leq l \\ 0 & \text { otherwise }\end{cases}
$$

Then, we obtain

$$
\left\langle\sigma E_{x}\right\rangle=\frac{1}{l} \int_{0}^{l} \sigma(x+\xi, y) E_{x}(x+\xi, y) d \xi=\frac{a \sigma_{2}}{l}\left\langle E_{x}\right\rangle_{a^{\prime}}
$$


where $\left\langle E_{x}\right\rangle_{a}$ is the average electric field intensity inside the ferromagnetic lamination, which is given by the equation

$$
\left\langle E_{x}\right\rangle_{a}=\frac{1}{a} \int_{0}^{a} E_{x}(x+\xi, y) d \xi
$$

Similarly, the average electric field intensity in the dielectric medium between the laminations is

$$
\left\langle E_{x}\right\rangle_{b}=\frac{1}{b} \int_{a}^{a+b} E_{x}(x+\xi, y) d \xi
$$

Applying the boundary condition for the average components $\left\langle E_{x}\right\rangle_{a}$ and $\left\langle E_{x}\right\rangle_{b}$, we can approximately write

$$
\varepsilon_{1}\left\langle E_{x}\right\rangle_{a}=\varepsilon_{2}\left\langle E_{x}\right\rangle_{b}
$$

On the other hand, the full average electric field intensity has the following form:

$$
\left\langle E_{x}\right\rangle=\frac{a}{l}\left\langle E_{x}\right\rangle_{a}+\frac{b}{l}\left\langle E_{x}\right\rangle_{b}
$$

Equations (2.12) and (2.13) form a system of equations which may be elementary solved with respect to the average $\left\langle E_{x}\right\rangle_{a}$. Substituting $\left\langle E_{x}\right\rangle_{a}$ into (2.9), we finally obtain approximately

$$
\left\langle\sigma E_{x}\right\rangle=\frac{\varepsilon_{2} a}{\varepsilon_{2} a+\varepsilon_{1} b} \sigma_{2}\left\langle E_{x}\right\rangle=\left\langle\sigma_{2}\right\rangle\left\langle E_{x}\right\rangle+\frac{b}{l} \frac{a\left(\varepsilon_{2}-\varepsilon_{1}\right)}{\varepsilon_{2} a+\varepsilon_{1} b} \sigma_{2}\left\langle E_{x}\right\rangle,
$$

where $\left\langle\sigma_{2}\right\rangle=a \sigma_{2} / l$. The rest of the correlation functions can be calculated similarly by using the appropriate boundary conditions between a lamination and the dielectric. As a result, we obtain, that after averaging, the medium 2 becomes anisotropic, and the permeability, permittivity, and electrical conductance of the laminated ferromagnetic core acquire tensor properties, that is, $\langle\sigma \mathbf{E}\rangle=\boldsymbol{\sigma} \cdot\langle\mathbf{E}\rangle,\langle\varepsilon \mathbf{E}\rangle=\boldsymbol{\varepsilon} \cdot\langle\mathbf{E}\rangle$, and $\langle\mu \mathbf{E}\rangle=\boldsymbol{\mu} \cdot\langle\mathbf{E}\rangle$, where

$$
\begin{gathered}
\sigma=\left(\begin{array}{ccc}
\frac{\varepsilon_{2} a}{\varepsilon_{2} a+\varepsilon_{1} b} \sigma_{2} & 0 & 0 \\
0 & \left\langle\sigma_{2}\right\rangle & 0 \\
0 & 0 & \left\langle\sigma_{2}\right\rangle
\end{array}\right), \quad \varepsilon=\left(\begin{array}{ccc}
\frac{\varepsilon_{1} \varepsilon_{2} l}{\varepsilon_{2} a+\varepsilon_{1} b} & 0 & 0 \\
0 & \left\langle\varepsilon_{2}\right\rangle & 0 \\
0 & 0 & \left\langle\varepsilon_{2}\right\rangle
\end{array}\right), \\
\boldsymbol{\mu}=\left(\begin{array}{ccc}
\frac{\mu_{1} \mu_{2} l}{\mu_{1} a+\mu_{2} b} & 0 & 0 \\
0 & \left\langle\mu_{2}\right\rangle & 0 \\
0 & 0 & \left\langle\mu_{2}\right\rangle
\end{array}\right) .
\end{gathered}
$$


Finally, for both mediums, the tensors $\sigma, \varepsilon$, and $\boldsymbol{\mu}$ can be represented in the forms

$$
\begin{gathered}
\boldsymbol{\sigma}=\langle\sigma\rangle \mathbf{I}+\left(\frac{b}{l}\right) \chi_{\sigma} \mathbf{Q}, \\
\boldsymbol{\varepsilon}=\langle\varepsilon\rangle \mathbf{I}+\left(\frac{b}{l}\right) \chi_{\varepsilon} \mathbf{Q}, \\
\boldsymbol{\mu}=\langle\mu\rangle \mathbf{I}+\left(\frac{b}{l}\right) \chi_{\mu} \mathbf{Q},
\end{gathered}
$$

where

(a) for medium $1,\langle\sigma\rangle=0,\langle\varepsilon\rangle=\varepsilon_{1},\langle\mu\rangle=\mu_{1}$ and $\chi_{\sigma}=\chi_{\varepsilon}=\chi_{\mu}=0$;

(b) for medium 2,

$$
\begin{gathered}
\langle\sigma\rangle=\left\langle\sigma_{2}\right\rangle, \quad\langle\varepsilon\rangle=\left\langle\varepsilon_{2}\right\rangle=\frac{\left(\varepsilon_{1} a+\varepsilon_{2} b\right)}{l}, \quad\langle\mu\rangle=\left\langle\mu_{2}\right\rangle=\frac{\left(\mu_{2} a+\mu_{1} b\right)}{l}, \\
\chi_{\sigma}=\frac{a\left(\varepsilon_{2}-\varepsilon_{1}\right)}{\varepsilon_{2} a+\varepsilon_{1} b} \sigma_{2}, \quad X_{\varepsilon}=-\frac{a\left(\varepsilon_{1}-\varepsilon_{2}\right)^{2}}{\varepsilon_{2} a+\varepsilon_{1} b}, \quad \chi_{\mu}=-\frac{a\left(\mu_{1}-\mu_{2}\right)^{2}}{\mu_{1} a+\mu_{2} b} .
\end{gathered}
$$

I is the identity matrix, and the tensor $\mathbf{Q}$ has the following form:

$$
\mathbf{Q}=\left(\begin{array}{lll}
1 & 0 & 0 \\
0 & 0 & 0 \\
0 & 0 & 0
\end{array}\right)
$$
form:

Then, after averaging, the system of Maxwell's equations (2.6) takes the following

$$
\begin{gathered}
\nabla \times\langle\mathbf{H}\rangle=\boldsymbol{\sigma} \cdot\langle\mathbf{E}\rangle+\langle\mathbf{j}\rangle, \\
\nabla \times\langle\mathbf{E}\rangle=-\boldsymbol{\mu} \cdot \frac{\partial\langle\mathbf{H}\rangle}{\partial t}, \\
\nabla(\boldsymbol{\varepsilon} \cdot\langle\mathbf{E}\rangle)=\langle\rho\rangle, \\
\nabla(\boldsymbol{\mu} \cdot\langle\mathbf{H}\rangle)=0, \\
\frac{\partial\langle\rho\rangle}{\partial t}+\nabla(\boldsymbol{\sigma} \cdot\langle\mathbf{E}\rangle)=0 .
\end{gathered}
$$




\section{The Zero-Order Perturbation Theory}

The perturbation theory is an asymptotic method of solving differential equations $[8,9]$. This method is commonly used in quantum mechanics $[11,12]$. In order to develop the perturbation theory for the system of (2.19), the relation $b / l \ll 1$ is taken as a small parameter. Then, the average electromagnetic field components should be expanded in a power series in $(b / l)$ as follows:

$$
\begin{aligned}
\langle\mathbf{E}\rangle & =\langle\mathbf{E}\rangle_{0}+\sum_{k=1}^{\infty}\left(\frac{b}{l}\right)^{k}\langle\mathbf{E}\rangle_{k^{\prime}} \\
\langle\mathbf{H}\rangle & =\langle\mathbf{H}\rangle_{0}+\sum_{k=1}^{\infty}\left(\frac{b}{l}\right)^{k}\langle\mathbf{H}\rangle_{k^{\prime}}
\end{aligned}
$$

where $\langle\mathbf{E}\rangle_{0}$ and $\langle\mathbf{H}\rangle_{0}$ are the leading terms of the series (3.1), $\langle\mathbf{E}\rangle_{k}$ and $\langle\mathbf{H}\rangle_{k}$ are the $k$-order terms of the perturbation theory. In this paper, we were limited only to the first order of the perturbation theory.

Substituting the expansions (3.1) into the system of (2.19), we obtain for the zero-order perturbation theory in the frequency domain

$$
\begin{gathered}
\nabla \times\langle\mathbf{H}\rangle_{0}=\langle\sigma\rangle\langle\mathbf{E}\rangle_{0}+\langle\mathbf{j}\rangle, \\
\nabla \times\langle\mathbf{E}\rangle_{0}=-j \omega\langle\mu\rangle\langle\mathbf{H}\rangle_{0}, \\
\nabla\left(\langle\varepsilon\rangle\langle\mathbf{E}\rangle_{0}\right)=\langle\rho\rangle_{0^{\prime}} \\
\nabla\left(\langle\mu\rangle\langle\mathbf{H}\rangle_{0}\right)=0, \\
j \omega\langle\rho\rangle_{0}+\nabla\left(\langle\sigma\rangle\langle\mathbf{E}\rangle_{0}\right)=0 .
\end{gathered}
$$

Equations (3.2)-(3.4) are similar to the Maxwell's equations for the case of the homogeneous ferromagnetic core with the core permeability $\left\langle\mu_{2}\right\rangle$, conductivity $\left\langle\sigma_{2}\right\rangle$ and permittivity $\left\langle\varepsilon_{2}\right\rangle$. This problem carries axial symmetry, which makes the use of the cylindrical coordinates appropriate. Maxwell's equations for coils on homogeneous ferromagnetic cores are solved in [1]. The complete solution of the system of (3.2)-(3.4) for the electric field intensity can be represented in the form

$$
\left\langle\widehat{E}_{\phi}\right\rangle_{0}=\left\langle\widehat{E}_{\phi 1}\right\rangle_{0} \theta(r-R)+\left\langle\widehat{E}_{\phi 2}\right\rangle_{0} \theta(R-r)
$$

where $\left\langle\widehat{E}_{\phi}\right\rangle_{0}$ is the Fourier-transform of the electric field intensity $\left\langle E_{\phi}\right\rangle_{0}$ with respect to the $z$-coordinate

$$
\left\langle\widehat{E}_{\phi}\left(r, k_{z}\right)\right\rangle_{0}=\int_{-\infty}^{+\infty}\left\langle E_{\phi}(t, r, z)\right\rangle_{0} e^{-j k_{z} z} d z
$$


The electric field intensities outside and inside the core, that is, $\left\langle\widehat{E}_{\phi 1}\right\rangle_{0}$ and $\left\langle\widehat{E}_{\phi 2}\right\rangle_{0^{\prime}}$ are given by the following equations:

$$
\begin{aligned}
& \left\langle\widehat{E}_{\phi 1}\right\rangle_{0}=-j \omega \mu_{1} r_{1}\left[\theta\left(r_{1}-r\right) I_{1}\left(k_{z} r\right) K_{1}\left(k_{z} r_{1}\right)+\theta\left(r-r_{1}\right) I_{1}\left(k_{z} r_{1}\right) K_{1}\left(k_{z} r\right)+K_{1}\left(k_{z} r\right) K_{1}\left(k_{z} r_{1}\right)\right. \\
& \left.\times \frac{I_{0}\left(k_{z} R\right)}{K_{0}\left(k_{z} R\right)} \frac{1-\left(\mu_{1} /\left\langle\mu_{2}\right\rangle\right)\left(\alpha I_{0}(\alpha R) I_{1}\left(k_{z} R\right) / k_{z} I_{1}(\alpha R) I_{0}\left(k_{z} R\right)\right)}{1+\left(\mu_{1} /\left\langle\mu_{2}\right\rangle\right)\left(\alpha I_{0}(\alpha R) K_{1}\left(k_{z} R\right) / k_{z} I_{1}(\alpha R) K_{0}\left(k_{z} R\right)\right)}\right] \\
& \times I_{\phi} \sum_{n=1}^{N} e^{-j k_{z} n \tau} \\
& \left\langle\widehat{E}_{\phi 2}\right\rangle_{0}=-j \omega \mu_{1}\left\langle\mu_{2}\right\rangle k_{z} r_{1} K_{1}\left(k_{z} r_{1}\right) I_{1}(\alpha r) \\
& \times \frac{I_{0}\left(k_{z} R\right) K_{1}\left(k_{z} R\right)+I_{1}\left(k_{z} R\right) K_{0}\left(k_{z} R\right)}{\mu_{1} \alpha I_{0}(\alpha R) K_{1}\left(k_{z} R\right)+\left\langle\mu_{2}\right\rangle k_{z} I_{1}(\alpha R) K_{0}\left(k_{z} R\right)} I_{\phi} \sum_{n=1}^{N} e^{-j k_{z} n \tau},
\end{aligned}
$$

where $\alpha^{2}=k_{z}^{2}+j \omega\left\langle\mu_{2}\right\rangle\left\langle\sigma_{2}\right\rangle$, and $I_{0}, I_{1}, K_{0}$ and $K_{1}$ are the well-known Bessel functions [13]. The difference between the solutions (3.5), (3.7), and (3.8) and the results obtained in [1] is that (3.5), (3.7), and (3.8) contain the averaged core parameters $\left\langle\mu_{2}\right\rangle$ and $\left\langle\sigma_{2}\right\rangle$, which are smaller than the ferromagnetic parameters $\mu_{2}$ and $\sigma_{2}$.

The solutions (3.5), (3.7), and (3.8) are going to be used in the solution of equations for the first-order perturbation theory.

\section{The First-Order Perturbation}

In the first-order perturbation theory, we obtain from the system of (2.19) in the frequency domain

$$
\begin{gathered}
\nabla \times\langle\mathbf{H}\rangle_{1}=\langle\sigma\rangle\langle\mathbf{E}\rangle_{1}+\chi_{\sigma} \mathbf{Q} \cdot\langle\mathbf{E}\rangle_{0}, \\
\nabla \times\langle\mathbf{E}\rangle_{1}=-j \omega\left(\langle\mu\rangle\langle\mathbf{H}\rangle_{1}+\chi_{\mu} \mathbf{Q} \cdot\langle\mathbf{H}\rangle_{0}\right), \\
\nabla\left(\langle\varepsilon\rangle\langle\mathbf{E}\rangle_{1}+\chi_{\varepsilon} \mathbf{Q} \cdot\langle\mathbf{E}\rangle_{0}\right)=\langle\rho\rangle_{1}, \\
\nabla\left(\langle\mu\rangle\langle\mathbf{H}\rangle_{1}+\chi_{\mu} \mathbf{Q} \cdot\langle\mathbf{H}\rangle_{0}\right)=0, \\
j \omega\langle\rho\rangle_{1}+\nabla\left(\langle\sigma\rangle\langle\mathbf{E}\rangle_{1}+\chi_{\sigma} \mathbf{Q} \cdot\langle\mathbf{E}\rangle_{0}\right)=0 .
\end{gathered}
$$

Equations (4.1) are equivalent to Maxwell's equations for a polarized medium with the electric current density $\mathbf{J}$, magnetization $\mathbf{M}$, and the polarization density $\mathbf{P}$, given by 
the following equations:

$$
\begin{gathered}
\mathbf{J}=\chi_{\sigma} \mathbf{Q} \cdot\langle\mathbf{E}\rangle_{0}=\chi_{\sigma} \mathbf{e}_{x}\left\langle E_{x}\right\rangle_{0}, \\
\mathbf{M}=\chi_{\mu} \mathbf{Q} \cdot\langle\mathbf{H}\rangle_{0}=\chi_{\mu} \mathbf{e}_{x}\left\langle H_{x}\right\rangle_{0}, \\
\mathbf{P}=\chi_{\varepsilon} \mathbf{Q} \cdot\langle\mathbf{E}\rangle_{0}=\chi_{\varepsilon} \mathbf{e}_{x}\left\langle E_{x}\right\rangle_{0},
\end{gathered}
$$

where $\mathbf{e}_{x}$ is the unit vector codirectional with the $X$ axis. These vector fields are calculated based on the solutions for the zero-order perturbation theory, and, therefore, in the first-order perturbation theory, they are considered as given functions. The contribution of the vectors $\mathbf{J}$ and $\mathbf{P}$ to the fields $\langle\mathbf{E}\rangle_{1}$ and $\langle\mathbf{H}\rangle_{1}$ can be taken into account in a future research.

From the definition of the coefficients $\chi_{\sigma}$ and $\chi_{\varepsilon}$ given above it follows, that in the case $\varepsilon_{1}=\varepsilon_{2}$, these two constants are zero, that is, $\chi_{\sigma}=\chi_{\varepsilon}=0$. As a result, the vector fields $\mathbf{J}$ and $\mathbf{P}$ become zeros, and therefore, the fields $\langle\mathbf{E}\rangle_{1}$ and $\langle\mathbf{H}\rangle_{1}$ are caused only by the magnetization M. Further, only this case, in which the permittivities $\varepsilon_{1}$ and $\varepsilon_{2}$ are very close or equal, will be analyzed.

Substituting $\mathbf{J}=\mathbf{P}=0$ into (4.1), we obtain

$$
\begin{gathered}
\nabla \times\langle\mathbf{H}\rangle_{1}=\langle\sigma\rangle\langle\mathbf{E}\rangle_{1}, \\
\nabla \times\langle\mathbf{E}\rangle_{1}=-j \omega\left(\langle\mu\rangle\langle\mathbf{H}\rangle_{1}+\mathbf{M}\right), \\
\nabla\left(\langle\varepsilon\rangle\langle\mathbf{E}\rangle_{1}\right)=\langle\rho\rangle_{1}, \\
\nabla\left(\langle\mu\rangle\langle\mathbf{H}\rangle_{1}+\mathbf{M}\right)=0, \\
j \omega\langle\rho\rangle_{1}+\nabla\left(\langle\sigma\rangle\langle\mathbf{E}\rangle_{1}\right)=0,
\end{gathered}
$$

where $\left\langle\varepsilon_{2}\right\rangle=\varepsilon_{1} \equiv \varepsilon_{0}$ is the vacuum permittivity. From (4.3) it follows that $\langle\rho\rangle_{1}=0$. Then, excluding the field $\langle\mathbf{H}\rangle_{1}$ from (4.3), we can obtain the equations for the electric field intensity $\langle\text { E }\rangle_{1}$ for mediums 1 and 2, respectively,

$$
\begin{gathered}
\Delta\left\langle\mathbf{E}_{1}\right\rangle_{1}=0, \\
\Delta\left\langle\mathbf{E}_{2}\right\rangle_{1}-j \omega\left\langle\mu_{2}\right\rangle\left\langle\sigma_{2}\right\rangle\left\langle\mathbf{E}_{2}\right\rangle_{1}=j \omega \nabla \times \mathbf{M} .
\end{gathered}
$$

Equation (4.4) is to be solved with the evident boundary condition at the core surface

$$
\begin{aligned}
& \left.\left\langle E_{\phi 1}\right\rangle_{1}\right|_{r=R}=\left.\left\langle E_{\phi 2}\right\rangle_{1}\right|_{r=R^{\prime}} \\
& \left.\left\langle H_{z 1}\right\rangle_{1}\right|_{r=R}=\left.\left\langle H_{z 2}\right\rangle_{1}\right|_{r=R^{\prime}} .
\end{aligned}
$$


The calculation of mutual impedance between the inducing coil and the filamentary turn, which is the aim of this paper, requires of the angular component $\left\langle E_{\phi 1}(r, z, \phi)\right\rangle$ of the intensity $\langle\mathbf{E}\rangle$, integrated over the turn of the radius $r[1]$, that is,

$$
Z=\frac{-\int_{0}^{2 \pi}\left\langle E_{\phi 1}(r, z, \phi)\right\rangle r d \phi}{I_{\phi}}=Z_{0}+\left(\frac{b}{l}\right) Z_{1}+\cdots
$$

where $Z_{0}$ is the mutual impedance in the zero-order approximation, which can be represented in the following form [1]:

$$
\begin{aligned}
Z_{0}=j \omega \mu_{1} r_{1} r \int_{-\infty}^{+\infty} d k_{z}[ & \theta\left(r_{1}-r\right) I_{1}\left(k_{z} r\right) K_{1}\left(k_{z} r_{1}\right) \\
& +\theta\left(r-r_{1}\right) I_{1}\left(k_{z} r_{1}\right) K_{1}\left(k_{z} r\right)+K_{1}\left(k_{z} r\right) K_{1}\left(k_{z} r_{1}\right) \\
& \left.\times \frac{I_{0}\left(k_{z} R\right)}{K_{0}\left(k_{z} R\right)} \frac{1-\left(\mu_{1} /\left\langle\mu_{2}\right\rangle\right)\left(\alpha I_{0}(\alpha R) I_{1}\left(k_{z} R\right) / k_{z} I_{1}(\alpha R) I_{0}\left(k_{z} R\right)\right)}{1+\left(\mu_{1} /\left\langle\mu_{2}\right\rangle\right)\left(\alpha I_{0}(\alpha R) K_{1}\left(k_{z} R\right) / k_{z} I_{1}(\alpha R) K_{0}\left(k_{z} R\right)\right)}\right] \\
\times &
\end{aligned}
$$

and $Z_{1}$ is the first-order perturbation of the whole mutual impedance. Let us denote the component $\left\langle E_{\phi 1}(r, z, \phi)\right\rangle_{1}$ of the electric field intensity $\langle\mathbf{E}\rangle_{1}$, averaged over all the directions $\phi$, as $\left\langle\left\langle E_{\phi 1}(r, z)\right\rangle\right\rangle$, where

$$
\left\langle\left\langle E_{\phi 1}(r, z)\right\rangle\right\rangle=\frac{1}{2 \pi} \int_{0}^{2 \pi}\left\langle E_{\phi 1}(r, z, \phi)\right\rangle_{1} d \phi
$$

Then, the first-order perturbation term $Z_{1}$ of the full mutual impedance can be calculated as follows:

$$
Z_{1}=-\frac{2 \pi r\left\langle\left\langle E_{\phi 1}(r, z)\right\rangle\right\rangle}{I_{\phi}}
$$

Let us apply the averaging procedure (4.8) to (4.4). The advantage of the use of the averaging (4.8) is that the equations for $\left\langle\left\langle E_{\phi}\right\rangle\right\rangle$ acquire axial symmetry, that is, this averaged component loses the dependence on the angle $\phi$. Then, after averaging procedure (4.8) and (4.4) can be written in the cylindrical coordinates as follows:

(a) for medium 1 ,

$$
\frac{1}{r} \frac{\partial}{\partial r}\left(r \frac{\partial\left\langle\left\langle E_{\phi 1}\right\rangle\right\rangle}{\partial r}\right)+\frac{\partial^{2}\left\langle\left\langle E_{\phi 1}\right\rangle\right\rangle}{\partial z^{2}}-\frac{\left\langle\left\langle E_{\phi 1}\right\rangle\right\rangle}{r^{2}}=0
$$


(b) for medium 2,

$$
\begin{aligned}
& \frac{1}{r} \frac{\partial}{\partial r}\left(r \frac{\partial\left\langle\left\langle E_{\phi 2}\right\rangle\right\rangle}{\partial r}\right)+\frac{\partial^{2}\left\langle\left\langle E_{\phi 2}\right\rangle\right\rangle}{\partial z^{2}}-\frac{\left\langle\left\langle E_{\phi 2}\right\rangle\right\rangle}{r^{2}}-j \omega\left\langle\mu_{2}\right\rangle\left\langle\sigma_{2}\right\rangle\left\langle\left\langle E_{\phi 2}\right\rangle\right\rangle \\
& \quad=j \omega \frac{1}{2 \pi} \int_{0}^{2 \pi} \mathbf{e}_{\phi}(\nabla \times \mathbf{M}) d \phi
\end{aligned}
$$

where the right-hand side of (4.11) can be easily calculated as follows:

$$
\frac{1}{2 \pi} \int_{0}^{2 \pi} \mathbf{e}_{\phi}(\nabla \times \mathbf{M}) d \phi=\frac{\chi_{\mu}}{2 \pi} \int_{0}^{2 \pi} \frac{\partial\left\langle H_{r 2}\right\rangle_{0}}{\partial z} \cos ^{2} \phi d \phi=\frac{\chi_{\mu}}{2} \frac{\partial\left\langle H_{r 2}\right\rangle_{0}}{\partial z} .
$$

The magnetic field in the zero-order perturbation theory in virtue of (3.3) can be obtained from the solution for medium 2 (3.8) in the form

$$
\left\langle H_{r 2}\right\rangle_{0}=-\frac{1}{j \omega\left\langle\mu_{2}\right\rangle} \frac{\partial\left\langle E_{\phi_{2}}\right\rangle_{0}}{\partial z} .
$$

Then, (4.10) and (4.11) become

$$
\begin{gathered}
\frac{\partial^{2}\left\langle\left\langle\widehat{E}_{\phi 1}\right\rangle\right\rangle}{\partial r^{2}}+\frac{1}{r} \frac{\partial\left\langle\left\langle\widehat{E}_{\phi 1}\right\rangle\right\rangle}{\partial r}-\left(k_{z}^{2}+\frac{1}{r^{2}}\right)\left\langle\left\langle\widehat{E}_{\phi 1}\right\rangle\right\rangle=0, \\
\frac{\partial^{2}\left\langle\left\langle\widehat{E}_{\phi 2}\right\rangle\right\rangle}{\partial r^{2}}+\frac{1}{r} \frac{\partial\left\langle\left\langle\widehat{E}_{\phi 2}\right\rangle\right\rangle}{\partial r}-\left(\alpha^{2}+\frac{1}{r^{2}}\right)\left\langle\left\langle\widehat{E}_{\phi 2}\right\rangle\right\rangle=\frac{x_{\mu} k_{z}^{2}}{2\left\langle\mu_{2}\right\rangle}\left\langle\widehat{E}_{\phi 2}\right\rangle_{0},
\end{gathered}
$$

where the Fourier transform $\left\langle\left\langle\widehat{E}_{\phi}\right\rangle\right\rangle$ of the field $\left\langle\left\langle E_{\phi}\right\rangle\right\rangle$ with respect to the $z$-coordinate is taken. Equations (4.14) and (4.15) are to be complemented with the averaged boundary conditions at the core surface

$$
\begin{aligned}
& \left.\left\langle\left\langle E_{\phi 1}\right\rangle\right\rangle\right|_{r=R}=\left.\left\langle\left\langle E_{\phi 2}\right\rangle\right\rangle\right|_{r=R^{\prime}} \\
& \left.\left\langle\left\langle H_{z 1}\right\rangle\right\rangle\right|_{r=R}=\left.\left\langle\left\langle H_{z 2}\right\rangle\right\rangle\right|_{r=R} .
\end{aligned}
$$

The solution of (4.14), taking into account the limit requirement $\left\langle\left\langle\widehat{E}_{\phi 1}\right\rangle\right\rangle \rightarrow 0$ as $r \rightarrow$ $\infty$, takes the following form:

$$
\left\langle\left\langle\widehat{E}_{\phi 1}\right\rangle\right\rangle=C_{1} K_{1}\left(k_{z} r\right)
$$


Equation (4.15) can be solved by means of the method of variation of constants. The solution of (4.15), after applying the condition $\left\langle\left\langle\widehat{E}_{\phi 2}\right\rangle\right\rangle \rightarrow 0$ as $r \rightarrow 0$, is the following:

$$
\begin{aligned}
\left\langle\left\langle\widehat{E}_{\phi 2}\right\rangle\right\rangle= & C_{2} I_{1}(\alpha r)+f\left(k_{z}\right) \\
\times & \left\{(\alpha r)^{2}\left(I_{1}^{2}(\alpha r)-I_{0}(\alpha r) I_{2}(\alpha r)\right)\left[I_{1}(\alpha r)+\frac{2 j}{\pi} K_{1}(\alpha r)\right]\right. \\
& \left.\quad-2 \alpha^{2} I_{1}(\alpha r) \int_{R}^{r} I_{1}(\alpha \eta)\left[I_{1}(\alpha \eta)+\frac{2 j}{\pi} K_{1}(\alpha \eta)\right] \eta d \eta\right\},
\end{aligned}
$$

where

$$
\begin{aligned}
f\left(k_{z}\right)= & -\frac{\pi \chi_{\mu} \omega \mu_{1} r_{1} k_{z}^{3}}{8 \alpha^{2}} K_{1}\left(k_{z} r_{1}\right) \\
& \times \frac{I_{0}\left(k_{z} R\right) K_{1}\left(k_{z} R\right)+I_{1}\left(k_{z} R\right) K_{0}\left(k_{z} R\right)}{\mu_{1} \alpha I_{0}(\alpha R) K_{1}\left(k_{z} R\right)+\left\langle\mu_{2}\right\rangle k_{z} I_{1}(\alpha R) K_{0}\left(k_{z} R\right)} I_{\phi} \sum_{n=1}^{N} e^{-j k_{z} n \tau} .
\end{aligned}
$$

The integral in the right-hand side of (4.18) can be calculated giving as a result the Meijer G-function [13]

$$
\begin{aligned}
& \int_{R}^{r} I_{1}(\alpha \eta)\left[I_{1}(\alpha \eta)+\frac{2 j}{\pi} K_{1}(\alpha \eta)\right] \eta d \eta \\
& \quad=\left.\frac{1}{2}\left(\eta^{2}\left[I_{1}^{2}(\alpha \eta)-I_{0}(\alpha \eta) I_{2}(\alpha \eta)\right]+\frac{j}{\pi^{3 / 2} \alpha^{2}} G_{24}^{22}\left(\begin{array}{c}
1,3 / 2 \\
1,2,0,0
\end{array} \mid(\alpha \eta)^{2}\right)\right)\right|_{R} ^{r}
\end{aligned}
$$

The constants $C_{1}, C_{2}$ are obtained from the boundary conditions (4.16). Substituting (4.17) and (4.18) into (4.16), we get

$$
\begin{aligned}
C_{1}= & -\frac{j \omega \chi_{\mu} \mu_{1}^{2} k_{z}^{2} r_{1}}{4} K_{1}\left(k_{z} r_{1}\right) \\
& \times \frac{I_{1}^{2}(\alpha R)-I_{0}(\alpha R) I_{2}(\alpha R)}{\left[\mu_{1} \alpha I_{0}(\alpha R) K_{1}\left(k_{z} R\right)+\left\langle\mu_{2}\right\rangle k_{z} I_{1}(\alpha R) K_{0}\left(k_{z} R\right)\right]^{2}} I_{\phi} \sum_{n=1}^{N} e^{-j k_{z} n \tau}
\end{aligned}
$$

and

$$
\begin{aligned}
C_{2}= & \frac{K_{1}\left(k_{z} R\right)}{I_{1}(\alpha R)} C_{1}-f\left(k_{z}\right)(\alpha R)^{2}\left(I_{1}(\alpha R)-\frac{I_{0}(\alpha R) I_{2}(\alpha R)}{I_{1}(\alpha R)}\right) \\
& \times\left(I_{1}(\alpha R)+\frac{2 j K_{1}(\alpha R)}{\pi}\right) .
\end{aligned}
$$


Substituting constant $C_{1}$ into (4.17) and then, the inverse Fourier transform of the field $\left\langle\left\langle\widehat{E}_{\phi 1}\right\rangle\right\rangle$ into (4.9), we obtain as a result the correction for the mutual impedance

$$
\begin{aligned}
Z_{1}= & \frac{j \omega \chi_{\mu} \mu_{1}^{2} r_{1} r}{4} \int_{-\infty}^{+\infty} d k_{z} k_{z}^{2} K_{1}\left(k_{z} r_{1}\right) K_{1}\left(k_{z} r\right) \\
& \times \frac{I_{1}^{2}(\alpha R)-I_{0}(\alpha R) I_{2}(\alpha R)}{\left[\mu_{1} \alpha I_{0}(\alpha R) K_{1}\left(k_{z} R\right)+\left\langle\mu_{2}\right\rangle k_{z} I_{1}(\alpha R) K_{0}\left(k_{z} R\right)\right]^{2}} \sum_{n=1}^{N} e^{j k_{z}(z-n \tau)} .
\end{aligned}
$$

Finally, the whole mutual impedance of coils on laminated ferromagnetic core is obtained by substituting (4.7) and (4.23) into (4.6).

Note that both terms $Z_{0}$ and $Z_{1}$ take into account the laminated structure of the core.

Formulas (4.6), (4.7), and (4.23) generalize the results of [1] in the sense that the result for the mutual impedance obtained in [1] is a particular case of the formulas obtained in the present paper.

The self impedance of a coil of $N$ turns of small cross section can be obtained from (4.6), (4.7), and (4.23) by taking $r$ and $z$ successively to coincide with the point on the surface of each turn of the coil and then summing the results for each turn of the coil. The self impedance of a single turn is a particular case which can be obtained by substituting $N=1$ into the formula for self impedance of a coil.

\section{Special Cases}

\subsection{Closed Cores}

An approximate formula for the mutual impedance for turns on a closed toroidal core can be obtained by placing the finite-length core between two infinite plates of perfect magnetic materials, that is, infinite permeability and conductance [1], with respective boundary conditions. In order to provide a reasonable accuracy to this approximation, the core is to be sufficiently long $(\tau \gg R)$, and the turns should be taken close to the core surface. In practice, these assumptions are equivalent to the case of an infinitely long core with an infinite number of energizing turns distributed periodically along the core surface [1]. The formulas (4.6), (4.7), and (4.23) are still applicable in this case, provided that the number of turns $N$ becomes infinite, $\tau$ is the closed core length, and the Fourier transform is to be replaced by a Fourier series, due to the periodical structure of the problem. Formally, the transition from the Fourier transform to the Fourier series in (4.7) and (4.23) can be fulfilled by substituting [1]

$$
\sum_{n=-\infty}^{+\infty} \exp \left(-j k_{z} n \tau\right)=\frac{2 \pi}{\tau} \sum_{k=-\infty}^{+\infty} \delta\left(k_{z}-\frac{2 \pi k}{\tau}\right)
$$

As a result, the formula for the complete mutual impedance takes the form

$$
Z=j \omega L_{A}+Z_{C 0}+\left(\frac{b}{l}\right) Z_{1}+\cdots
$$




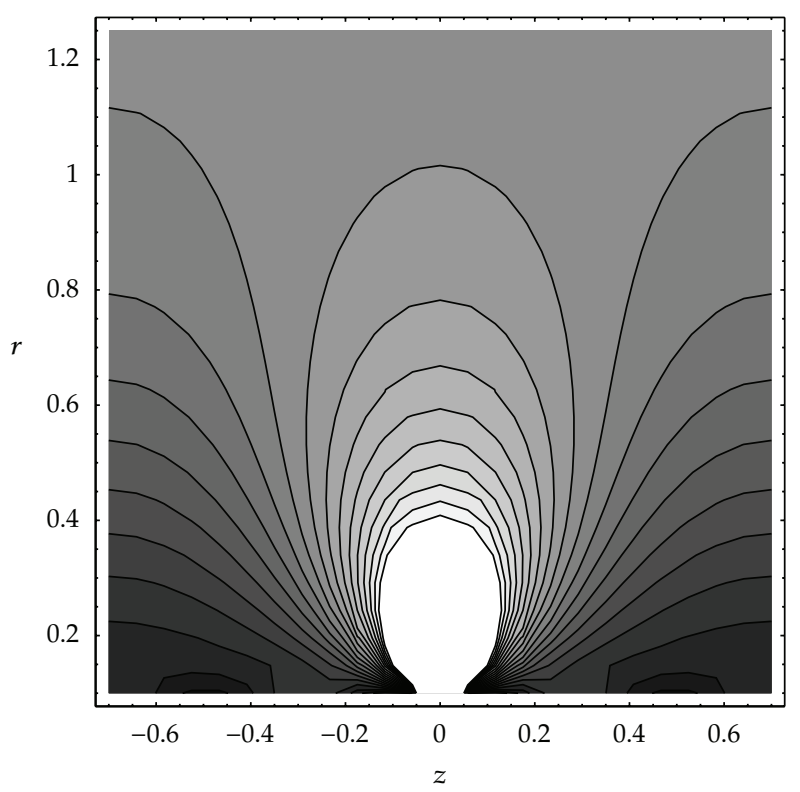

Figure 3: Contours of constant $L_{m}$ at $1 \mathrm{MHz}$.

where

$$
L_{A}=\mu_{1} r_{1} r \frac{2 \pi}{\tau} \sum_{k=-\infty}^{+\infty}\left[\theta\left(r_{1}-r\right) I_{1}\left(\beta_{k} r\right) K_{1}\left(\beta_{k} r_{1}\right)+\theta\left(r-r_{1}\right) I_{1}\left(\beta_{k} r_{1}\right) K_{1}\left(\beta_{k} r\right)\right] e^{j \beta_{k} z}
$$

is the inductance in air [1] and

$$
\begin{aligned}
Z_{C 0}= & j \omega \mu_{1} r_{1} r \frac{2 \pi}{\tau} \sum_{k=-\infty}^{+\infty} K_{1}\left(\beta_{k} r\right) K_{1}\left(\beta_{k} r_{1}\right) \\
& \times \frac{I_{0}\left(\beta_{k} R\right)}{K_{0}\left(\beta_{k} R\right)} \frac{1-\left(\mu_{1} /\left\langle\mu_{2}\right\rangle\right)\left(\alpha_{k} I_{0}\left(\alpha_{k} R\right) I_{1}\left(\beta_{k} R\right) / k_{z} I_{1}\left(\alpha_{k} R\right) I_{0}\left(\beta_{k} R\right)\right)}{1+\left(\mu_{1} /\left\langle\mu_{2}\right\rangle\right)\left(\alpha I_{0}\left(\alpha_{k} R\right) K_{1}\left(\beta_{k} R\right) / k_{z} I_{1}\left(\alpha_{k} R\right) K_{0}\left(\beta_{k} R\right)\right)} e^{j \beta_{k} z}, \\
Z_{1}= & \frac{j \omega \chi_{\mu} \mu_{1}^{2} r_{1} r}{4} \frac{2 \pi}{\tau} \sum_{k=-\infty}^{+\infty} \beta_{k}^{2} K_{1}\left(\beta_{k} r_{1}\right) K_{1}\left(\beta_{k} r\right) \\
& \times \frac{I_{1}^{2}\left(\alpha_{k} R\right)-I_{0}\left(\alpha_{k} R\right) I_{2}\left(\alpha_{k} R\right)}{\left[\mu_{1} \alpha I_{0}\left(\alpha_{k} R\right) K_{1}\left(\beta_{k} R\right)+\left\langle\mu_{2}\right\rangle k_{z} I_{1}\left(\alpha_{k} R\right) K_{0}\left(\beta_{k} R\right)\right]^{2}} e^{j \beta_{k} z},
\end{aligned}
$$

and $\beta_{k}=2 \pi k / \tau$ and $\alpha_{k}^{2}=\beta_{k}^{2}+j \omega\left\langle\mu_{2}\right\rangle\left\langle\sigma_{2}\right\rangle$.

In Figures 3 and 4 , contours of constant $L_{m}=\operatorname{Im} Z(\omega) / \omega$ are presented for different frequencies and as a function of $z$ and $r$. For illustrative purposes, the following parameters values have been taken: $\sigma=1.667 \times 10^{6} \mathrm{~S} / \mathrm{m}, \mu_{1}=\mu_{0}, \mu_{2}=4045 \mu_{0}, R=0.1 \mathrm{~m}, r_{1}=0.11 \mathrm{~m}$, $\tau=1.4 \mathrm{~m}, b=106 \mu \mathrm{m}$, and $a=248 \mu \mathrm{m}$. Figure 3 represents contours of constant $L_{m}(\omega)$ at the frequency $1 \mathrm{MHz}$. Figure 4 illustrates contours of constant inductive part of the mutual 


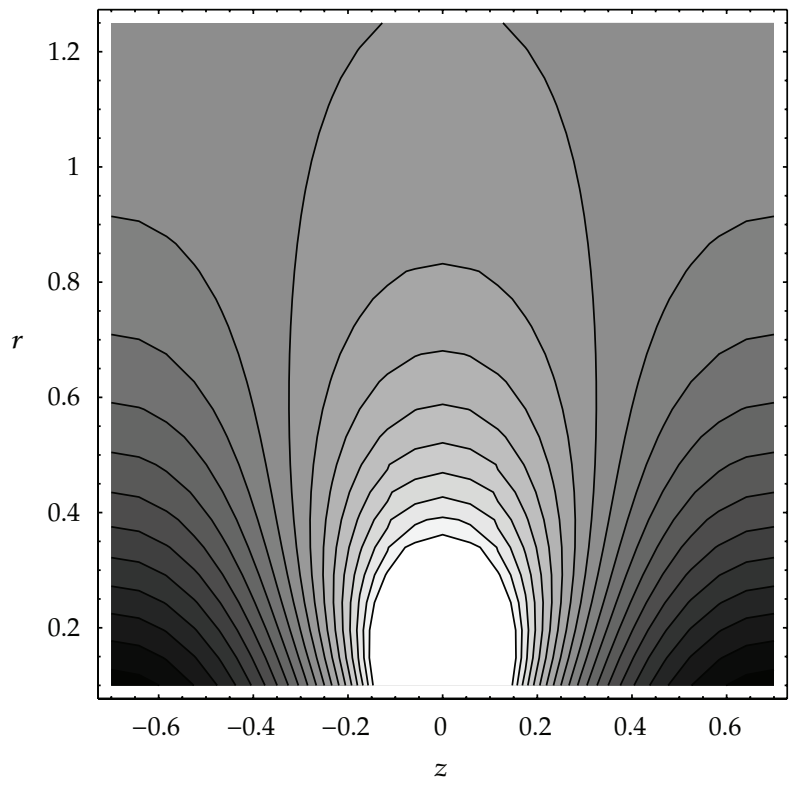

Figure 4: Contours of constant $L_{m}$ at $60 \mathrm{~Hz}$.

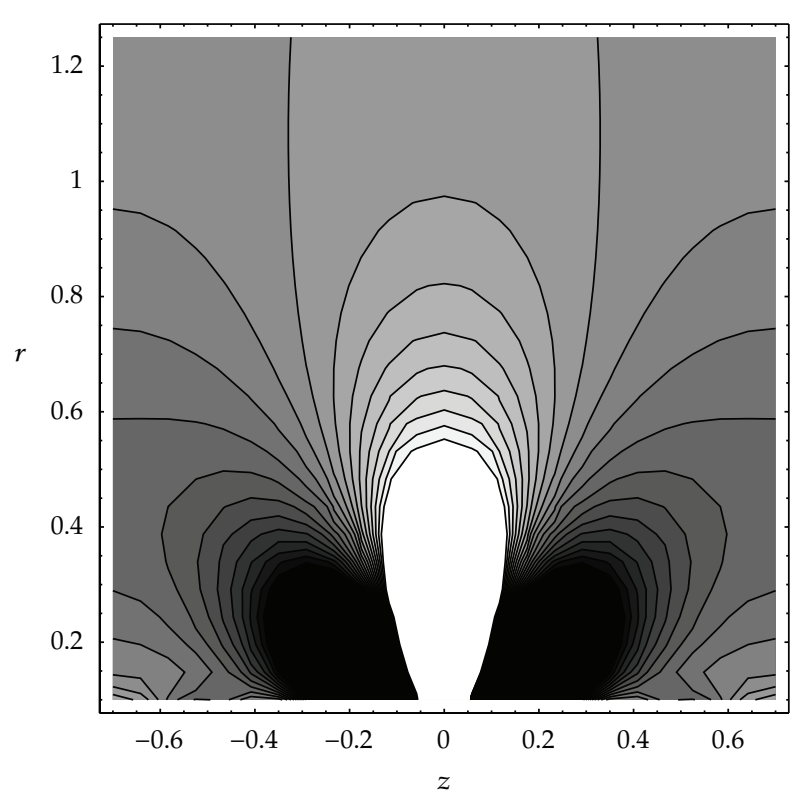

Figure 5: Contours of constant $\operatorname{Re} Z_{1}$ at $1 \mathrm{MHz}$.

impedance at $\omega=2 \pi 60 \mathrm{rad} / \mathrm{s}$. The mutual inductance $L_{m}(\omega)$ increases from the dark parts to the light regions. Figures 3 and 4 , respectively, illustrate the effects of different ranges of flux penetration into the core. The behavior of the resistive part $R_{m}=\operatorname{Re} Z(\omega)$ of the mutual impedance is somewhat similar, and therefore, the respective figures have been omitted. 


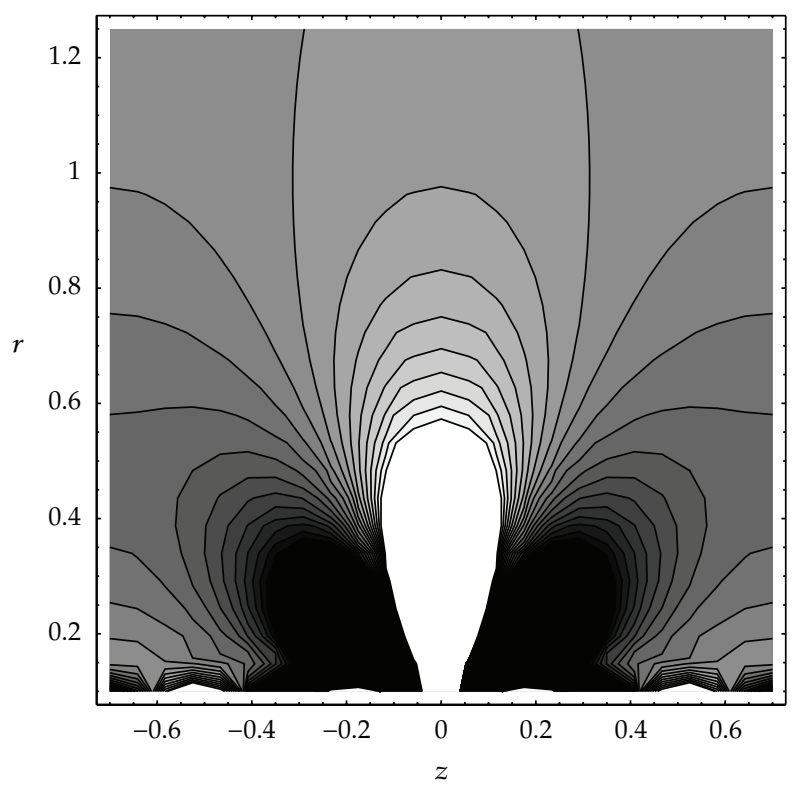

Figure 6: Contours of constant $\operatorname{Im} Z_{1} / \omega$ at $1 \mathrm{MHz}$.

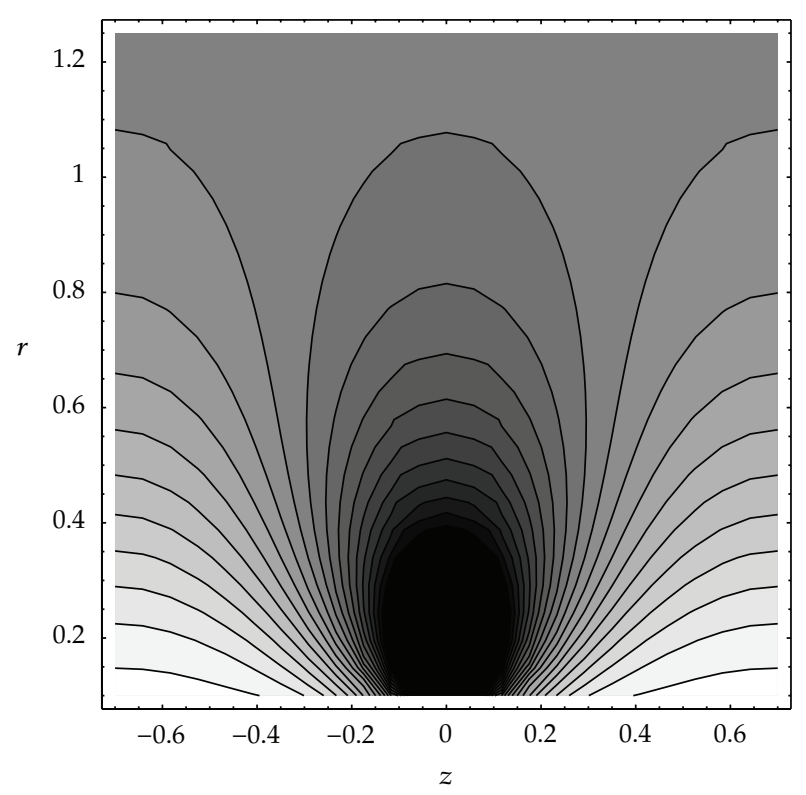

Figure 7: Contours of constant $\operatorname{Re} Z_{1}$ at $60 \mathrm{~Hz}$.

In Figures 5, 6, 7, and 8, the behavior of the correction term $Z_{1}$ at different frequencies is presented. At $60 \mathrm{~Hz}$, the resistive part of the correction impedance $Z_{1}$ brings a negative contribution to the complete mutual impedance near the energizing filamentary turn, whereas at $1 \mathrm{MHz}$ it is positive. 


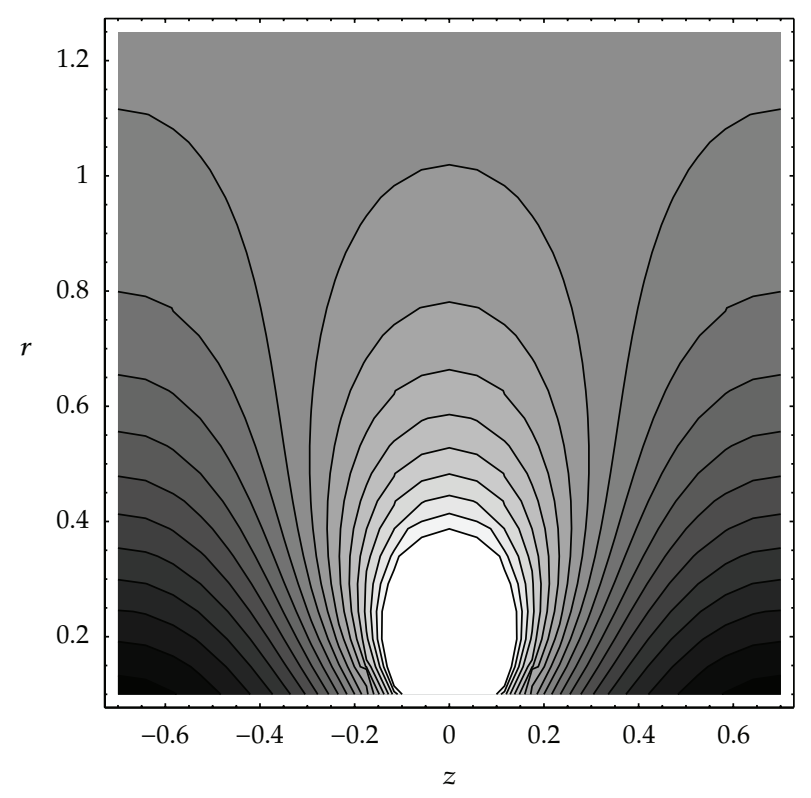

Figure 8: Contours of constant $\operatorname{Im} Z_{1} / \omega$ at $60 \mathrm{~Hz}$.

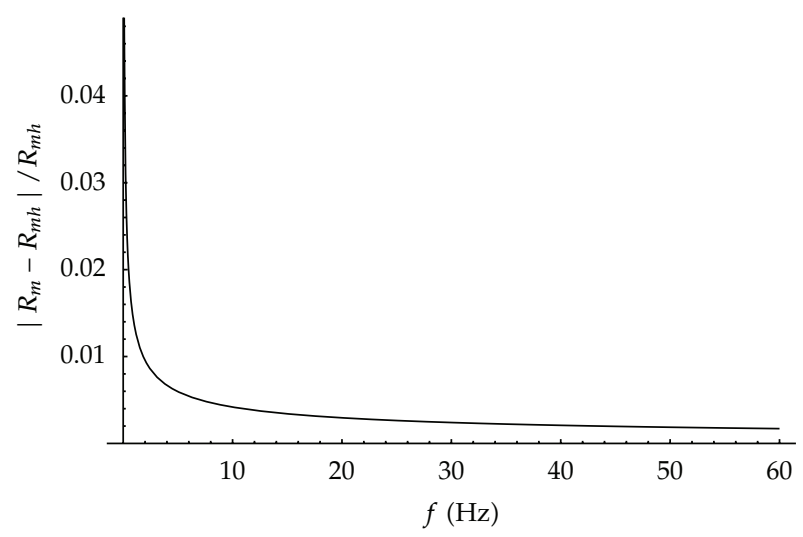

Figure 9: $\left|R_{m}-R_{m h}\right| / R_{m h}$ curve versus frequency.

From Figures 3 and 4 and [1], we can see that the behavior of the mutual impedance of turns on homogeneous and laminated ferromagnetic cores is somewhat similar. In order to compare the mutual impedance of coils on a laminated ferromagnetic core $L_{m}$ and on a homogeneous ferromagnetic core $L_{m h}$, and to estimate the maximum contribution of the laminated core to the mutual impedance, let us plot the relative increment of the mutual impedance and resistance. The core parameters are the same as those in Figures 3-8.

In Figure 9, the $\left|R_{m}-R_{m h}\right| / R_{m h}$ curve versus the frequency is shown at $r=0.1112 \mathrm{~m}$ and $z=0.7 \mathrm{~m}$. A maximum difference (up to $66 \%$ ) under the conditions mentioned above occurs at the limit $\omega \rightarrow 0$. 
In Figure 10, the $\left(L_{m}-L_{m h}\right) / L_{m h}$ curve at $r=0.1112 \mathrm{~m}$ and $z=0.7 \mathrm{~m}$ is shown. The maximum contribution $(17.5 \%)$ of the laminated structure of the core, for the given conditions, occurs at approximately $16.5 \mathrm{kHz}$.

\subsection{Coils on an Infinite Core}

The mutual and self impedance for coils on an infinite core can be obtained under the same assumptions that are given in [1]. We consider two coils of the radius $r_{1}$ and $r_{2}$, widths $w_{1}$ and $w_{2}$, thicknesses $h_{1}$ and $h_{2}$, and the turn numbers $N_{1}$ and $N_{2}$. The distance between two coils is $z$. Then, the mutual impedance caused by the laminated ferromagnetic core is the following:

$$
\begin{aligned}
Z_{C}= & Z_{C 0}+\left(\frac{b}{l}\right) Z_{1}=\frac{2 N_{1} N_{2}}{h_{1} h_{2} w_{1} w_{2}} \\
& \times \int_{0}^{\infty} P_{1}\left(k_{z}\left(r_{1}+h_{1}\right), k_{z} r_{1}\right) P_{1}\left(k_{z}\left(r_{2}+h_{2}\right), k_{z} r_{2}\right) \\
& \times Q_{1}\left(k_{z} w_{2}, k_{z} w_{1}\right) G\left(k_{z}\right) \cos \left(k_{z} z\right) d k_{z},
\end{aligned}
$$

where

$$
\begin{gathered}
G\left(k_{z}\right)=j \omega \mu_{1}\left[\frac{I_{0}\left(k_{z} R\right)}{K_{0}\left(k_{z} R\right)} \frac{1-\left(\mu_{1} /\left\langle\mu_{2}\right\rangle\right)\left(\alpha I_{0}(\alpha R) I_{1}\left(k_{z} R\right) / k_{z} I_{1}(\alpha R) I_{0}\left(k_{z} R\right)\right)}{1+\left(\mu_{1} /\left\langle\mu_{2}\right\rangle\right)\left(\alpha I_{0}(\alpha R) K_{1}\left(k_{z} R\right) / k_{z} I_{1}(\alpha R) K_{0}\left(k_{z} R\right)\right)}\right. \\
\left.+\frac{b}{l} \frac{x_{\mu} \mu_{1} k_{z}^{2}}{4} \frac{I_{1}^{2}(\alpha R)-I_{0}(\alpha R) I_{2}(\alpha R)}{\left[\mu_{1} \alpha I_{0}(\alpha R) K_{1}\left(k_{z} R\right)+\left\langle\mu_{2}\right\rangle k_{z} I_{1}(\alpha R) K_{0}\left(k_{z} R\right)\right]^{2}}\right], \\
P_{1}(x, y)=\frac{1}{k_{z}^{2}}\left[p_{1}(x)-p_{2}(y)\right], \\
Q_{1}(x, y)=\frac{4}{k_{z}^{2}} \sin \left(\frac{x}{2}\right) \sin \left(\frac{y}{2}\right), \\
p_{1}(x)=\frac{\pi x}{2}\left(K_{1}(x) L_{0}(x)+K_{0}(x) L_{1}(x)\right),
\end{gathered}
$$

where $L_{v}(x)$ is the modified Struve function [13]

$$
L_{v}(x)=\sum_{m=0}^{\infty}\left(\frac{x}{2}\right)^{v+2 m+1} \frac{1}{\Gamma(m+3 / 2) \Gamma(v+m+3 / 2)} .
$$

In the case of the self impedance calculations of a coil on an infinite ferromagnetic laminated core we should put: $r_{1}=r_{2}, w_{1}=w_{2}, h_{1}=h_{2}$ and $N_{1}=N_{2}$. 


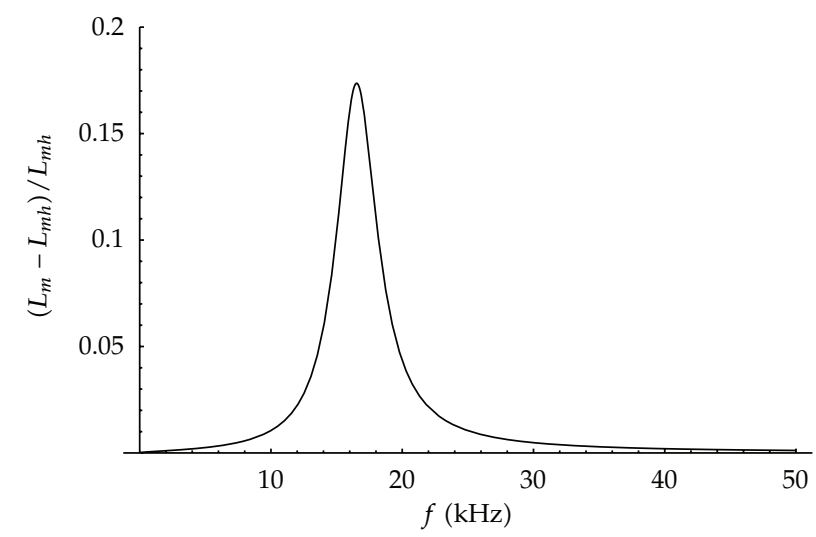

Figure 10: $\left(L_{m}-L_{m h}\right) / L_{m h}$ curve versus frequency.

\subsection{Coils on a Closed Core}

For the case of coils on a closed core, we can fulfill the same considerations that have been done in the previous section. Then, the formula for the mutual impedance for two coils on a ferromagnetic laminated toroidal core takes the form

$$
Z=j \omega L_{A}+Z_{C}
$$

where

$$
\begin{aligned}
& Z_{C}=\frac{\pi}{\tau} N_{1} N_{2}\{C+\frac{4}{h_{1} h_{2} w_{1} w_{2}} \sum_{k=1}^{\infty} Q_{1}\left(\beta_{k} w_{2}, \beta_{k} w_{1}\right) \\
&\left.\times P_{1}\left(\beta_{k}\left(r_{1}+h_{1}\right), \beta_{k} r_{1}\right) P_{1}\left(\beta_{k}\left(r_{2}+h_{2}\right), \beta_{k} r_{2}\right) G\left(\beta_{k}\right) \cos \left(\beta_{k} z\right)\right\}, \\
& C=j \omega R^{2}\left(\frac{2\left\langle\mu_{2}\right\rangle I_{1}\left(\sqrt{j \omega\left\langle\mu_{2}\right\rangle\left\langle\sigma_{2}\right\rangle} R\right)}{\sqrt{j \omega\left\langle\mu_{2}\right\rangle\left\langle\sigma_{2}\right\rangle} R I_{0}\left(\sqrt{j \omega\left\langle\mu_{2}\right\rangle\left\langle\sigma_{2}\right\rangle} R\right)}-\mu_{1}\right) .
\end{aligned}
$$

In Figures 11 and 12, the theoretical curves and experimental points for the resistive (Figure 11) and inductive (Figure 12) components of self impedance in the frequency range $10^{2} \mathrm{~Hz}$ to $1 \mathrm{MHz}$ for test coil on an outer limb of the $25 \mathrm{kVA}$ core are presented. The experimental data and the core and coil parameters were taken from [3]: $\sigma=97.0874 \Omega^{-1}$, $a=0.27 \mu \mathrm{m}, b=0.02 \mu \mathrm{m}, \mu_{1}=\mu_{0}, \mu_{2}=843 \mu_{1}, \tau=0.976 \mathrm{~m}, R=0.047 \mathrm{~m}$, and the test core has 12 turns of heavy-gauge wire, and so forth. The inductance in air is calculated following the recommendations given in [3]. Both theoretical curves show a good agreement between measurements and calculations, which confirms once again the idea expressed in [3] about the validity of treating the laminated core as solid.

Also, in [3] it has been expressed that in spite of a good accuracy of obtained formulas, the prediction of transient behavior of transformer under general conditions requires some 


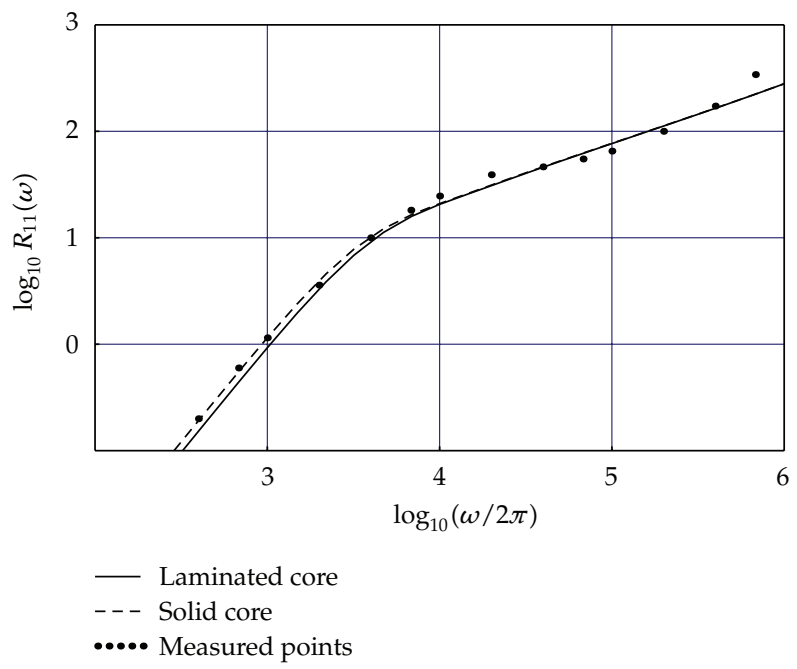

Figure 11: Resistive component of self impedance (test coil on an outer limb of the $25 \mathrm{kVA}$ core). Dashed line corresponds to the solid core and the continuous line represents the laminated core.

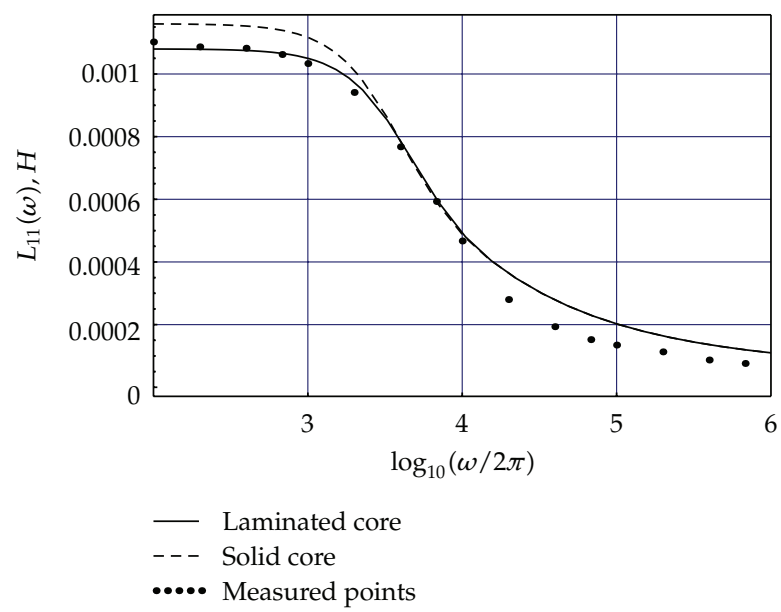

Figure 12: Inductive component of self impedance (test coil on an outer limb of the $25 \mathrm{kVA}$ core). Dashed line corresponds to the solid core and the continuous line represents the laminated core.

more accurate considerations. In particular, the consideration of the differential mutual impedance $\Delta Z(\omega)=Z_{11}(\omega)-Z_{12}(\omega)=\Delta R(\omega)+j \omega \Delta L(\omega)$ is required. Any substantial errors obtained in the calculated quantities $\Delta R(\omega)$ and $\Delta L(\omega)$ for the solid core (dotted lines in Figures 13 and 14) may result in significant errors in transient oscillations. Therefore, it is important to keep these errors small.

To improve these results, it was proposed in [3] to enhance the core resistivity. This proposal seems reasonable because this is that really occurs inside the core. Indeed, the averaged conductivity, that is, presented in (5.9), is smaller than the conductivity of lamination, that is, $\left\langle\sigma_{2}\right\rangle=a \sigma_{2} / l<\sigma_{2}$, and therefore, the core resistivity increases. 


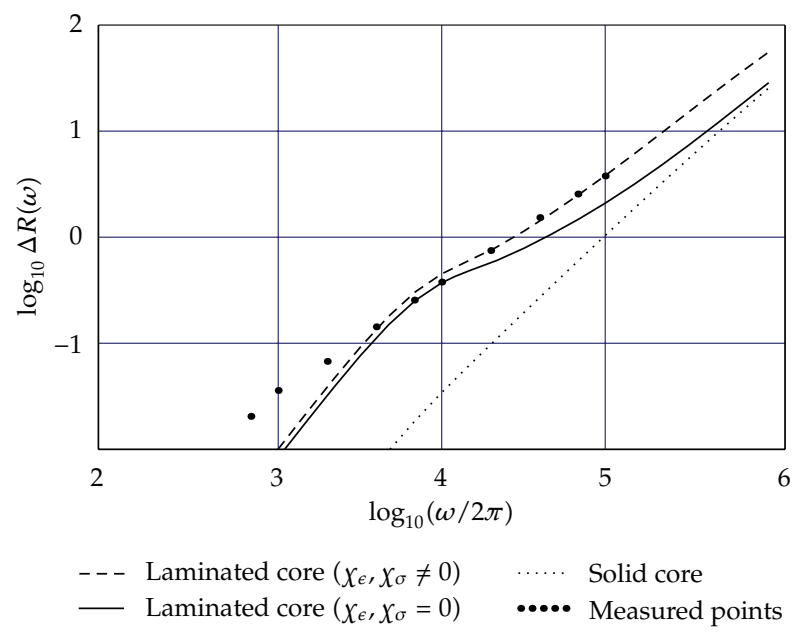

Figure 13: Resistive component of differential impedance between two test coils (coils on outer limb of the $25 \mathrm{kVA}$ core at separation of $0.2 \mathrm{~m}$ ). Dotted line corresponds to the solid core, the continuous line represents the laminated core with one parameter calculated $\chi_{\mu}$ and the dashed line is for two calculated parameters $X_{\mu}$ and $\chi_{\varepsilon}$.

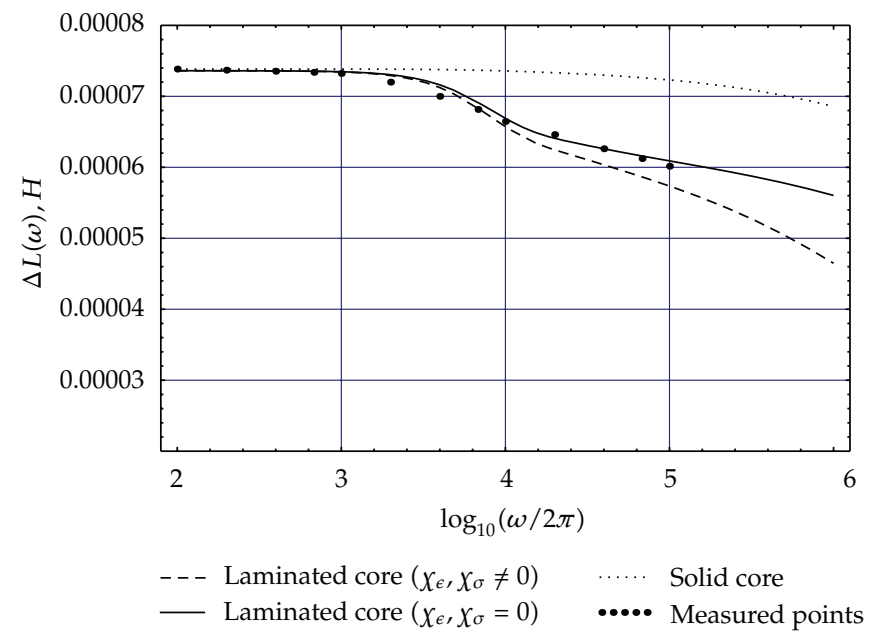

Figure 14: Inductive component of differential impedance between two test coils (coils on outer limb of the $25 \mathrm{kVA}$ core at separation of $0.2 \mathrm{~m}$ ). Dotted line corresponds to the solid core, the continuous line represents the laminated core with one parameter calculated $\chi_{\mu}$ and the dashed line is for two calculated parameters $x_{\mu}$ and $\chi_{\varepsilon}$.

Nevertheless, on the contrary, a simple numerical analysis shows that the reduction of the averaged conductivity results in a larger error between the theoretical and experimental results. To corroborate this, it is enough to substitute $\chi_{\mu}=0$ in (5.6) and plot the differential resistivity and inductance curves. Therefore, a simple change of the core resistivity cannot improve the agreement between the theoretical and experimental results. 
On the other hand, taking into account the first-order perturbative term, that is, assuming $\chi_{\mu} \neq 0$, improves the theoretical results only a little. A reasonable explanation of this fact can lie in how the correlations $\langle\sigma \mathbf{E}\rangle,\langle\varepsilon \mathbf{E}\rangle$, and $\langle\mu \mathbf{H}\rangle$ have been calculated. The correct calculation of these correlations implies having on hand the solutions for $\mathbf{E}$ and $\mathbf{H}$ before solving Maxwell's equations, which is impossible. Then, there are two possible ways to deal with these terms. Both methods consider the parameters $\chi_{\sigma}, \chi_{\varepsilon}$, and $\chi_{\mu}$ as unknown constants. In the first one, these parameters should be recalculated after solving the exact Maxwell's equations. The second way is to calculate these constants by comparing the theoretical and the experimental results. However, the first way is not feasible in this particular case because the technique proposed in this paper has the purpose of avoiding the necessity of calculating the exact fields $\mathbf{E}$ and $\mathbf{H}$. Then, the second program should be carried out.

In Figures 13 and 14, the theoretical and experimental behavior of the differential resistivity and inductance, respectively, is presented. The dotted line shows the theoretical behavior of the differential impedance in the case of the solid core [3]. The continuous line represents the theoretical differential impedance for the laminated core with the parameter $x_{\mu}$ calculated to obtain a good agreement with the experimental differential inductance. In this case, to obtain the parameter $x_{\mu}$, one experimental point as a reference is enough. However, to obtain the best result, the least squares method can be used. The obtained result is $X_{\mu}=32.06 \mathrm{H}$, and the theoretical result obtained with this parameter value shows an excellent agreement with the experimental data in the case of the differential inductance, and a better approximation for the differential resistance.

In turn, in this method, the rest of parameters, that is, $\chi_{\varepsilon}$ and $\chi_{\sigma}$ cannot be taken as zero, and therefore, the impedance terms corresponding to the vectors $\mathbf{J}$ and $\mathbf{P}$, cannot be neglected. Figures 13 and 14 show the differential resistivity and inductance calculated (the dashed line), taking into account the terms $\mathbf{J}$ and $\mathbf{P}$ in the first-order perturbation theory (the corresponding contribution of these terms to the impedance is calculated similarly as the contribution corresponding to the term $\mathbf{M}$ ). The parameters $\chi_{\mu}=33.24 \mathrm{H}, \chi_{\varepsilon}=$ $-1.30282 \times 10^{-9} \mathrm{~F} / \mathrm{m}$, and $\chi_{\sigma}=4764.1 \Omega^{-1}$ are calculated to obtain a better correlation with the experimental data by using the least squares method. We can see that, involving the vectors $\mathbf{J}$ and $\mathbf{P}$ into the differential impedance calculation, we obtain a better approximation of the theoretical differential resistivity to the measured data, especially in the high frequency range.

In [3], it was proposed to adjust the theoretical results with measurements by introducing an additional empiric term in the formula for the differential impedance. On the contrary, the formulas obtained in this paper are theoretical and completely consistent with Maxwell's equations. Therefore, it seems more logical and consistent to compare our results with the experimental data and the respective theoretical results for the solid core [3], which has been carried out in this section.

\section{Conclusions}

New formulas for calculating the self and mutual impedances of coils on laminated ferromagnetic cores of circular cross-section have been derived. These formulas generalize the previously established formulas for the homogeneous ferromagnetic cores [1], for the case of the laminated cores. In the limit $b \rightarrow 0$ and $a \rightarrow l$, the obtained formulas are transformed into the well-known formulas for the self and mutual impedances for coils on homogeneous ferromagnetic cores published in [1]. 
The obtained result for the frequency-dependent self and mutual impedances for coils on laminated core is compared with the experimental and theoretical results for coils on a practical transformer core, published in [3]. The presented numerical results show a very good correspondence of the new formulas to the measured data. These new formulas do not require introducing the additional empiric terms in order to obtain a better correspondence to the experimental points; all the new terms, involved in the impedance formula, are fully consistent with Maxwell's equations. Moreover, the accuracy, reached in the new formulas, is not the limit; it can be improved by a more thin analysis of the correlation terms $\langle\sigma \mathbf{E}\rangle,\langle\varepsilon \mathbf{E}\rangle$, and $\langle\mu \mathbf{H}\rangle$. Thus, all the stated above can be considered as a method for further theoretical research.

As the proposed method implies the strict solving of Maxwell's equations, it can be used not only for impedance calculation, but also for the electromagnetic fields analysis inside the core. For example, substituting (4.22) into (4.18), we can obtain the averaged electric field intensity inside the core, which, in turn, can be used in the analysis of the laminated core heating. In turn, it calls for numerical evaluation of different special functions such as the Meijer G-function [13]. However, with available computers and established numerical techniques, this is no longer a problem [1]. The programming of the Meijer G-function is not a great obstacle; this function can be calculated with any required degree of accuracy, which is not particularly time consuming.

Further theoretical work could be done to establish the exact formulas for the toroidal cores and cores of different geometries. Also, it is of interest the theoretical analysis of the thermal effects occurring inside the laminated core during the transformer operation. The proposed methods, taken as a principle for solving this problem, make possible such type of analysis.

\section{Acknowledgments}

This paper was supported by the Dirección General de Educación Superior Tecnológica and the Consejo Nacional de Ciencia y Tecnología-Sistema Nacional de Investigadores of México.

\section{References}

[1] D. J. Wilcox, M. Conlon, and W. G. Hurley, "Calculation of self and mutual impedances for coils on ferromagnetic cores," IEE Proceedings A, vol. 135, no. 7, pp. 470-476, 1988.

[2] J. Avila-Rosales and F. L. Alvarado, "Nonlinear frequency dependent transformer model for electromagnetic transient studies in power systems," IEEE Transactions on Power Apparatus and Systems, vol. 101, no. 11, pp. 4281-4288, 1982.

[3] D. J. Wilcox, W. G. Hurley, and M. Conlon, "Calculation of self and mutual impedances between sections of transformer windings," IEE Proceedings C, vol. 136, no. 5, pp. 308-314, 1989.

[4] H. Akçay and D. G. Ece, "Modeling of hysteresis and power losses in transformer laminations," IEEE Transactions on Power Delivery, vol. 18, no. 2, pp. 487-492, 2003.

[5] S. Maximov and V. H. Coria, Métodos analíticos en el cálculo de impedancias de transformadores con diferentes núcleos, M.S. thesis, Programa de Graduados en Investigación en Ingeniería Eléctrica, México, Mexico, 2008.

[6] J. H. Harlow, Electric Power Transformer Engineering, IEEE, CRC Press LLC, Boca Raton, Fla, USA, 2004.

[7] L. L. Grigsby, Electric Power Engineering Handbook, IEEE, CRC Press LLC, Boca Raton, Fla, USA, 1998.

[8] M. Reed and B. SaĬmon, Methods of Modern Mathematical Physics, Academic Press, San Diego, Calif, USA, 1978.

[9] J. Mathews and R. L. Walker, Mathematical Methods of Physics, Addison-Wesley, Redwood City, Calif, USA, 2nd edition, 1969. 
[10] V. S. Vladimirov, Generalized Functions in Mathematical Physics, Mir, Moscow, Russia, 1979.

[11] L. I. Schiff, Quantum Mechanics, McGraw-Hill, New York, NY, USA, 2nd edition, 1965.

[12] L. D. Landau, M. D. Lifshitz, and L. P. Pitaevskii, Electrodynamics of Continuos Media, U.S.S.R. Academy of Sciences, Moscow, Russia, 2nd edition, 1984.

[13] L. C. Andrews, Special Functions for Engineers and Applied Mathematicians, Macmillan, New York, NY, USA, 1985. 


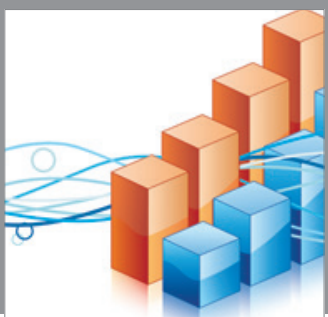

Advances in

Operations Research

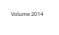

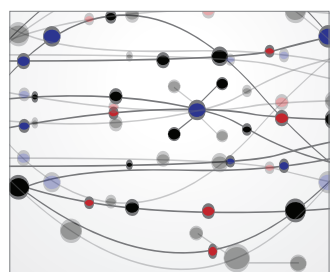

\section{The Scientific} World Journal
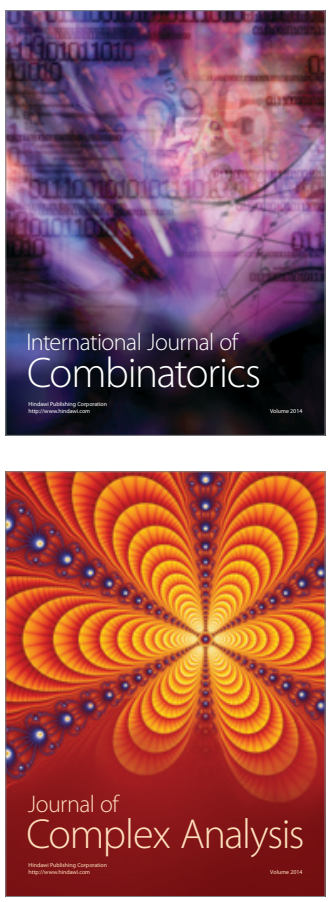

International Journal of

Mathematics and

Mathematical

Sciences
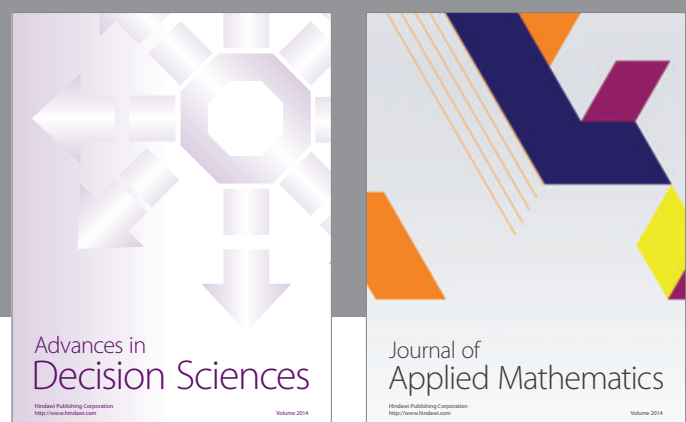

Journal of

Applied Mathematics
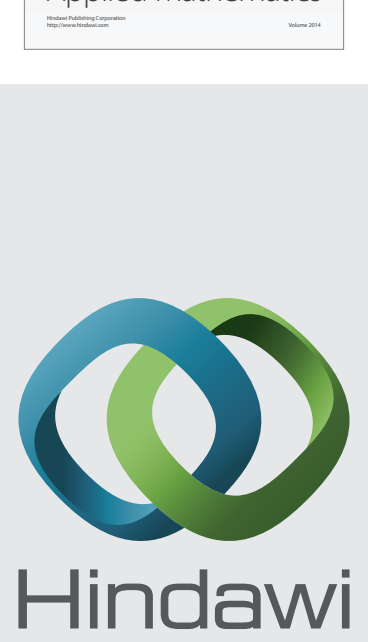

Submit your manuscripts at http://www.hindawi.com
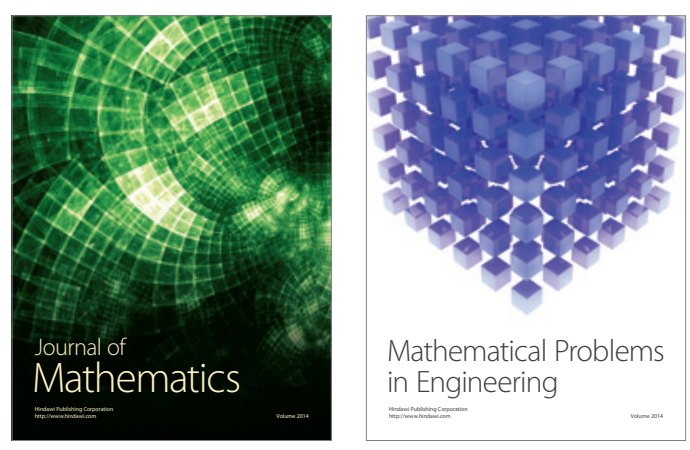

Mathematical Problems in Engineering
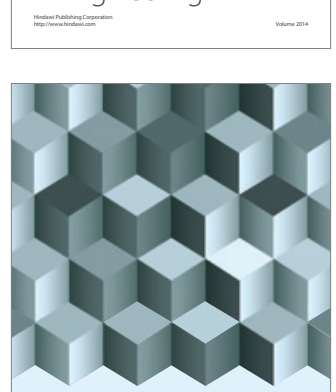

Journal of

Function Spaces
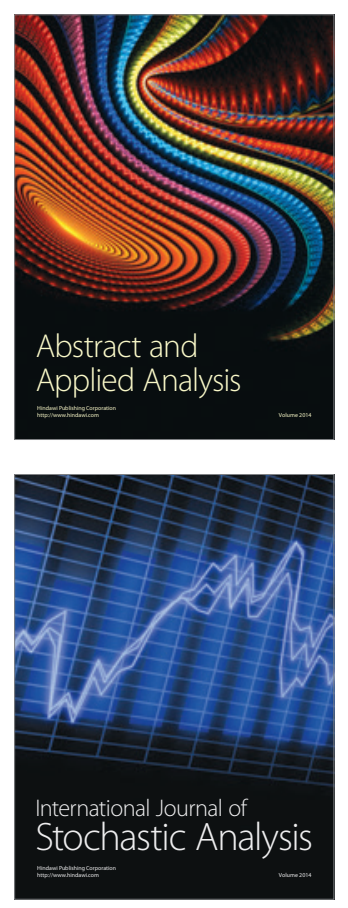

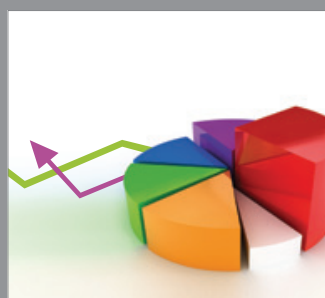

ournal of

Probability and Statistics

Promensencen
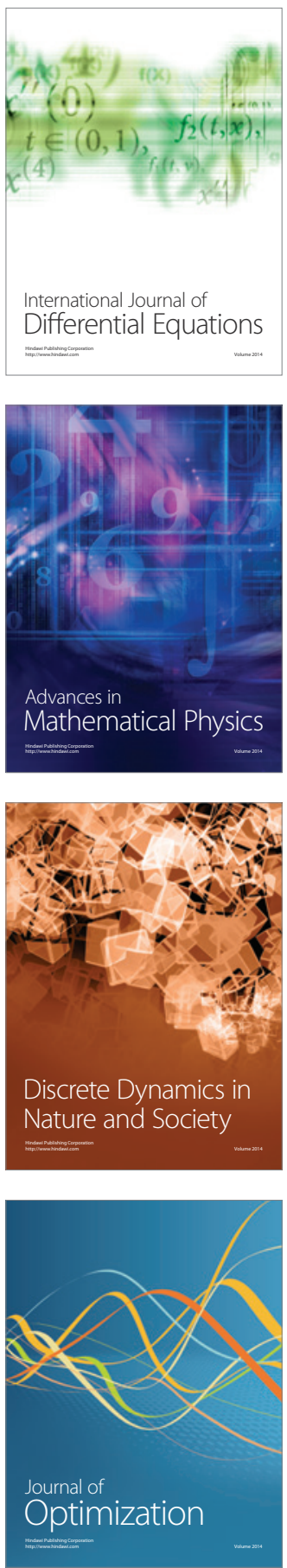